Working Paper

Business Economic Series

WP. 18-03 November, 2018

ISSN 1989-8843
Instituto para el Desarrollo de Empresas y

Mercados.

Universidad Carlos III de Madrid

C/ Madrid, 126

28903 Getafe Madrid (Spain)

http://indem.uc3m.es/en/index

http://indem.uc3m.es/en/workingpapers

\title{
Golden options in financial mathematics
}

\author{
Alejandro Balbás ${ }^{1}$ \\ Departamento de Economía de la Empresa \\ Universidad Carlos III de Madrid \\ Beatriz Balbás² \\ Departamento de Economía y Dirección de Empresas \\ Universidad de Alcalá de Henares \\ Raquel Balbás ${ }^{3}$ \\ Departamento Economía Financiera y Métodos de Decisión. \\ Universidad Complutense de Madrid
}

\footnotetext{
${ }^{1}$ C/ Madrid, 126. 28903 Getafe (Madrid).

2 PI. de la Victoria, 2. 28802 Alcalá de Henares (Madrid).

${ }^{3}$ Campus de Somosaguas. 28223 Pozuelo de Alarcón (Madrid).
} 


\title{
Golden options in financial mathematics
}

\author{
Alejandro Balbás*, Beatriz Balbás** and Raquel Balbás***
}

* University Carlos III of Madrid. C/ Madrid, 126. 28903 Getafe (Madrid, Spain). alejandro.balbas@uc3m.es

** University of Alcalá de Henares. Pl. de la Victoria, 2. 28802 Alcalá de Henares (Madrid, Spain). beatriz.balbas@uah.es

*** University Complutense of Madrid. Somosaguas. 28223 Pozuelo de Alarcón (Madrid, Spain).raquel.balbas@ccee.ucm.es

A.M.S. 91G10, 91G20, 91G80, 91B06.

J.E.L. G11, G13, C61, C65.

Key-words Golden option, Risk measure, Smooth good deal, Dual approach.

Abstract. This paper deals with the construction of "smooth good deals" (SGD), i.e., sequences of self-financing strategies whose global risk diverges to $-\infty$ and such that every security in every strategy of the sequence is a "smooth" derivative with a bounded delta.

If the selected risk measure is the value at risk then these sequences exist under quite weak conditions, since one can involve risks with both bounded and unbounded expectation, as well as non-friction-free pricing rules. Moreover, every strategy in the sequence is composed of an European option plus a position in a riskless asset. The strike of the option is easily computed in practice, and the ideas may also apply in some actuarial problems such as the selection of an optimal reinsurance contract.

If the chosen risk measure is a coherent one then the general setting is more limited. Indeed, though frictions are still accepted, expectations and variances must remain finite. The existence of SGDs will be characterized, and computational issues will be properly addressed as well. It will be shown that SGDs often exist, and for the conditional value at risk they are composed of the riskless asset plus easily replicable European puts. Numerical experiments will be presented in all of the studied cases.

\section{Introduction}

Since Artzner et al. (1999) introduced the coherent risk measures in their seminal paper, there has been a growing interest in performance/risk measurement. Among many other significant contributions, there are generalized deviations (Rockafellar et al., 2006), dynamic time-consistent risk measures (Kupper and Schachermayer, 2009), set-valued risk measures (Jouini, et al., 2004), exhaustive distortion risk measure (Balbás, et al., 2009), conditional risk measures (Filipović, et al., 2012), natural risk measures (Assa, 2016), gain-loss ratios (Bernardo and Ledoit ,2000), omega ratios (Mausser et al., 2006), etc.

When dealing with $V @ R$ or a coherent risk measure such as $C V @ R$, it is not unusual to find strategies whose risk in a long (short) position is lower than the risk of a (short) riskless asset with the same price. Thus, if a trader 
borrows (lends) the strategy price and buys (sells) it, he/she will create a selffinancing portfolio with negative risk. Moreover, if one deals with $C V @ R$, the expected pay-off of this portfolio is strictly positive. Hence, if the whole strategy can be implemented once and once again, with no limit, one will be facing a sequence of self-financing investment strategies whose expected pay-off diverges to $+\infty$ and whose risk diverges to $-\infty{ }^{1}$ A similar drawback has been found by Biagini and Pinar (2013) for the gain-loss ratio. They show that the Black and Scholes $(B S)$ model and other continuous time pricing models theoretically allow traders to replicate sequences of strategies whose gain-loss ratio diverges to $+\infty$. The authors argue that these strategies in the sequence contain many digital options, which are too difficult to replicate in practice due to the delta instability. Consequently, they conclude that the best gain-loss ratio may be a poor performance measure in a continuous time framework, since the possibility of an unbounded gain-loss ratio is more theoretical than real.

Balbás et al. (2016) have created sequences of self-financing strategies whose (expected_pay-off, $V @ R, C V @ R)$ theoretically diverges to $(+\infty,-\infty,-\infty)$ and have studied the empirical performance of these strategies in several international future markets. The main conclusion is that the strategies always outperform the underlying future contract, ${ }^{2}$ though the divergence to $(+\infty,-\infty,-\infty)$ does not hold. A major caveat in the analysis of Balbás et al. (2016) is that their strategies always contain digital options that they cannot replicate, and consequently the empirical test has to focus on "approximations" of the "ideal optimal strategy" obtained in the theoretical part of the paper.

This paper deals with both $V @ R$ and a coherent risk measure $\rho$, and answers the Question_1 below:

Question_1. Can one construct "smooth enough" strategies whose risk (risk when short) is lower than the risk of a (short) riskless asset with the same price?

In particular, Section 2 focuses on $V @ R$, while Sections 3 and 4 focus on a coherent measure of risk. The meaning of "smooth enough" will be clear, since we will only deal with a portfolio of derivatives whose delta at maturity cannot be larger than one.

As said above, we first focus on $V @ R$. Though $V @ R$ presents some theoretical shortcomings, it is still a very popular risk measure often used by practitioners, regulators, supervisors and researchers. ${ }^{3}$ Moreover, $V @ R$ is the unique usual risk measure remaining finite when dealing with heavy tailed risks with unbounded expectation. Our analysis may involve both bounded and unbounded expectations, as well as transaction costs in the pricing rule. Theorem 2 is the most important result of the second section, and it shows that the answer to Question_1 is very often "yes", despite the fact that we are facing a quite general framework. Furthermore, the desired strategy is really simple, since it is composed of the sale of an European option (the $V @ R$ "golden option",

\footnotetext{
${ }^{1}$ The existence of this sequence holds in many (arbitrage-free) pricing models whose selffinancing strategies become martingales under a risk-neutral measure, i.e., pricing models which are compatible with the existence of equilibrium (Jarrow and Larsson, 2012).

${ }^{2}$ similar results have been found in Konstantinides and Zachos (2018).

${ }^{3}$ See, for instance, Zhao and Xiao (2016) for a recent theoretical analysis involving $V @ R$.
} 
or $\left.V @ R \_G O\right)$. The option strike is easy to estimate in practice, and some numerical experiments are reported with the $B S$ model.

Section 3 replaces $V @ R$ with a coherent risk measure $\rho$. The framework has to be more limited. Indeed, expectations and variances have to remain finite, and $\rho$ must be continuous, but the pricing rule may still incorporate potential frictions. Theorem 18, Corollary 19 and their remarks characterize those cases such that the answer to Question_1 is "yes", and also show that this positive answer frequently holds. Theorem 21 supposes that $\rho=C V @ R$ and gives an explicit construction of the strategy we are interested in, which often equals the sale of a "golden European put" (the $C V @ R_{-} G P$ ). Some numerical experiments are reported.

Section 4 particularizes the analysis for discrete models. Though computational issues have been already addressed in Section 3, discrete models can be very easily treated with finite-dimensional linear programming methods, and therefore this case may deserve attention too. Actually, usual pricing models often have a good enough discrete approximation. The fifth section concludes the paper.

\section{$2 \quad \mathrm{~V} @ \mathrm{R}$ and golden options}

Consider $-\infty \leq a<b \leq+\infty$ and a probability space $((a, b), \mathcal{B}, \mathbb{P}), \mathcal{B}$ being the Borel $\sigma$-algebra of $(a, b)$ and $\mathbb{P}$ being a probability measure. We will consider that $\mathbb{P}$ is generated by $S_{T},{ }^{4}$ which is the potential wealth at a future date $T$ generated by some actuarial or financial position. As usual, let $L^{0}$ be the space of $\mathbb{R}$-valued measurable functions on $(a, b)$ and,$^{5}$ for $1 \leq p<\infty$ (respectively, $p=\infty$ ), let $L^{p}$ be the subspace of $L^{0}$ containing those $y \in L^{0}$ such that $\mathbb{E}\left(|y|^{p}\right)<\infty$ (respectively, Ess_Sup $(|y|)<\infty$ ), $\mathbb{E}($.$) denoting mathematical$ expectation (respectively, Ess_Sup denoting essential supremum). Let $Y \subset L^{0}$ be a convex cone of $L^{0}$. Consider a function $\Pi: Y \rightarrow \mathbb{R}$ representing the current price $\Pi(y)$ of every wealth $y \in Y$, and suppose that $\Pi$ is sub-additive, i.e.,

$$
\Pi\left(y+y^{\prime}\right) \leq \Pi(y)+\Pi\left(y^{\prime}\right)
$$

for every $y, y^{\prime} \in Y$, positively homogeneous, i.e.,

$$
\Pi(\kappa y)=\kappa \Pi(y)
$$

for $\kappa>0$ and $y \in Y$, and strictly increasing, i.e.,

$$
\Pi(y)>\Pi\left(y^{\prime}\right)
$$

if $\mathbb{P}\left(y-y^{\prime} \geq 0\right)=1$ and $\mathbb{P}\left(y-y^{\prime}>0\right)>0$. Obviously, $S_{T}$ can be identified with the identity map $(a, b) \ni x \rightarrow I(x)=x \in(a, b)$ and must belong to $Y$.

\footnotetext{
${ }^{4}$ i.e., $\mathbf{P}(B)=\mathbf{P}\left(S_{T} \in B\right)$ for every $B \in \mathcal{B}$.

${ }^{5}$ if necessary, we will denote $L^{0}(a, b)$ or $L^{0}((a, b), \mathcal{B}, \mathbf{P})$. Similar notations will apply in similar cases.
} 
Suppose also that the riskless asset belongs to $Y(k \in Y$ for every $k \in \mathbb{R})$, and denote by $r \leq R$ the lending rate and the borrowing rate, respectively, i.e.,

$$
\Pi(k)=k e^{-r T} \quad \text { and } \quad \Pi(-k)=-k e^{-R T} .
$$

for every $k \in \mathbb{R}, k \geq 0$. If $r=R$ then $r$ will be said to be the riskless rate. Proposition 1 below is not new, though we will give the proof in order to facilitate the reader's understanding.

Proposition 1 a) $\Pi(0)=0$, and (2) also holds if $\kappa=0$.

b) If $r=R$ then $\Pi(-k)=-\Pi(k)$ holds for every $k \in \mathbb{R}$.

c) If $r=R$ then $\Pi(y+k)=\Pi(y)+\Pi(k)$ holds for every $y \in Y$ and every $k \in \mathbb{R}$.

d) If $r=R$ then $\Pi\left(y-e^{r T} \Pi(y)\right)=\Pi\left(-y+e^{r T} \Pi(y)\right)=0$ holds for every $y \in Y$.

e) If $\mathbb{P}(y \geq 0)=1$ then $\Pi\left(-y-e^{r T} \Pi(-y)\right) \leq 0$.

Proof. a) Obviously, $\Pi(0)=\Pi(2 \times 0)=2 \Pi(0)$. Thus, $\Pi(0) \neq 0$ would lead to the contradiction $1=2$.

b) It trivially follows from (4).

c) For $y \in Y$ and $k \in \mathbb{R}$ one has that

$$
\Pi(y)=\Pi\left(\frac{y+k+y-k}{2}\right) \leq \frac{1}{2}(\Pi(y)+\Pi(k))+\frac{1}{2}(\Pi(y)-\Pi(k))=\Pi(y) .
$$

Hence, every inequality in the chain must become an equality.

d) (4) and c) lead to $\Pi\left(y-e^{r T} \Pi(y)\right)=\Pi(y)-\Pi\left(e^{r T} \Pi(y)\right)=\Pi(y)-$ $\Pi(y)=0$. Besides, taking $\left.y=-y^{\prime}, b\right)$ and $c$ ) lead to $\Pi\left(-y+e^{r T} \Pi(y)\right)=$ $\Pi\left(y^{\prime}-e^{r T} \Pi\left(y^{\prime}\right)\right)=0$.

e) If $\mathbb{P}(y \geq 0)=1$ then $\Pi\left(-y-e^{r T} \Pi(-y)\right) \leq \Pi(-y)+\Pi\left(-e^{r T} \Pi(-y)\right)=$ $\Pi(-y)-\Pi(-y)=0$.

Next, let us present a main result.

Theorem 2 Suppose that $\beta \in(0,1)$ is a confidence level, and take $k_{\beta}, K_{\beta} \in$ $(a, b)$.

a) If $\mathbb{P}\left(a, k_{\beta}\right)=1-\beta, r=R$ and the put option $\left(k_{\beta}-S_{T}\right)^{+}$belongs to $Y$ then $y=-\left(k_{\beta}-S_{T}\right)^{+}+e^{r T} \Pi\left(\left(k_{\beta}-S_{T}\right)^{+}\right)$satisfies $\Pi(y)=0$ and

$$
V @ R_{\beta}(y) \leq-e^{r T} \Pi\left(\left(k_{\beta}-S_{T}\right)^{+}\right)<0 .
$$

b) If $\mathbb{P}\left(a, k_{\beta}\right)=1-\beta$ and the put option $\left(k_{\beta}-S_{T}\right)^{+}$belongs to $Y$ then $y=-\left(k_{\beta}-S_{T}\right)^{+}-e^{r T} \Pi\left(-\left(k_{\beta}-S_{T}\right)^{+}\right)$satisfies $\Pi(y) \leq 0$ and $V @ R_{\beta}(y) \leq$ $e^{r T} \Pi\left(-\left(k_{\beta}-S_{T}\right)^{+}\right)<0$. 
c) If $\mathbb{P}\left(K_{\beta}, b\right)=1-\beta, r=R$ and the call option $\left(S_{T}-K_{\beta}\right)^{+}$belongs to $Y$ then $y=-\left(S_{T}-K_{\beta}\right)^{+}+e^{r T} \Pi\left(\left(S_{T}-K_{\beta}\right)^{+}\right)$satisfies $\Pi(y)=0$ and

$$
V @ R_{\beta}(y) \leq-e^{r T} \Pi\left(\left(S_{T}-K_{\beta}\right)^{+}\right)<0 .
$$

d) If $\mathbb{P}\left(K_{\beta}, b\right)=1-\beta$ and the call option $\left(S_{T}-K_{\beta}\right)^{+}$belongs to $Y$ then $y=-\left(S_{T}-K_{\beta}\right)^{+}-e^{r T} \Pi\left(-\left(S_{T}-K_{\beta}\right)^{+}\right)$satisfies $\Pi(y) \leq 0$ and $V @ R_{\beta}(y) \leq$ $e^{r T} \Pi\left(-\left(S_{T}-K_{\beta}\right)^{+}\right)<0$.

Proof. a) $\Pi(y)=0$ trivially follows from Proposition $1 d$. Let us prove (5). Consider the indicator $\chi_{\left(a, k_{\beta}\right)}$ of the interval $\left(a, k_{\beta}\right)$ and take $z=\chi_{\left(a, k_{\beta}\right)} /(1-\beta) \in$ $L^{\infty}, \lambda_{m}=0 \in Y, \lambda_{M}=\left(k_{\beta}-S_{T}\right)^{+} \in Y$ and $\lambda=-e^{r T} \Pi\left(\left(k_{\beta}-S_{T}\right)^{+}\right) \in \mathbb{R}$. Then,

$$
\left\{\begin{array}{l}
y=\lambda_{m}-\lambda_{M}-\lambda \\
\mathbb{E}(z)=1 \\
\lambda_{m} z=\lambda_{M}\left(\frac{1}{1-\beta}-z\right)=0 \\
z \leq 1 /(1-\beta) \\
z, \lambda_{m}, \lambda_{M} \geq 0
\end{array}\right.
$$

are obvious, and the $V @ R$ representation theorem of Balbás et al. (2017) leads to $V @ R_{\beta}(y) \leq \lambda=-e^{r T} \Pi\left(\left(k_{\beta}-S_{T}\right)^{+}\right)$. It only remains to see the inequality $\Pi\left(\left(k_{\beta}-S_{T}\right)^{+}\right)>0$, which trivially follows from (3).

b) $\Pi(y) \leq 0$ trivially follows from Proposition 1e. Besides, take $z, \lambda_{m}$ and $\lambda_{M}$ as above, and $\lambda=e^{r T} \Pi\left(-\left(k_{\beta}-S_{T}\right)^{+}\right) .(7)$ holds again and $V @ R_{\beta}(y) \leq$ $e^{r T} \Pi\left(-\left(S_{T}-K_{\beta}\right)^{+}\right)$. Moreover, $\Pi\left(-\left(S_{T}-K_{\beta}\right)^{+}\right)<0$ trivially follows from (3).

$c$ ) and $d$ ) are analogous to $a$ ) and $b$ ).

Henceforth $\mathbb{L}$ will denote the Lebesgue measure in $(a, b)$.

Remark 3 If $\mathbb{P}$ is equivalent to $\mathbb{L}$ then $\mathbb{P}$ is obviously atomless, and therefore both equations

$$
\mathbb{P}(a, k)=1-\beta
$$

and

$$
\mathbb{P}(K, b)=1-\beta
$$

will have a solution $k_{\beta}$ and $K_{\beta}$ for every $0<\beta<1$ (Lyapunov's theorem about the range of a $\sigma$-additive measure).

Remark 4 Suppose that $r=R . y=-\left(k_{\beta}-S_{T}\right)^{+}+e^{r T} \Pi\left(\left(k_{\beta}-S_{T}\right)^{+}\right)$is the wealth generated by the sale of the European put $\left(k_{\beta}-S_{T}\right)^{+}$jointly with the 
investment of the put price in the riskless asset. The positive homogeneity of $\Pi$ (see (2)) and $V @ R_{\beta}$ imply that

$$
n y=-n\left(k_{\beta}-S_{T}\right)^{+}+e^{r T} \Pi\left(n\left(k_{\beta}-S_{T}\right)^{+}\right)
$$

satisfies

$$
\left\{\begin{array}{l}
\Pi(n y)=0, \\
\lim _{n} V @ R_{\beta}(n y)=-\infty
\end{array} \quad \forall n \in \mathbb{N}\right.
$$

i.e., if the sale of the put $\left(k_{\beta}-S_{T}\right)^{+}$plus the investment in the riskless asset may be implemented as many times as desired, then agents can construct strategies whose price always vanishes and whose $V @ R$ becomes as negative as desired. For this reason $\left(k_{\beta}-S_{T}\right)^{+}$will be said to be the $V @ R$ golden put $\left(V @ R \_G P\right)$. Similarly, $\left(S_{T}-K_{\beta}\right)^{+}$will be said to be the $V @ R$ golden call $\left(V @ R \_G \bar{C}\right)$.

If $r<R$ then take $y=-\left(k_{\beta}-S_{T}\right)^{+}-e^{r T} \Pi\left(-\left(k_{\beta}-S_{T}\right)^{+}\right)$and (10) still holds if its first equality is replaced by $\Pi(n y) \leq 0$. Similar comments apply for the $V @ R_{-} G C$.

Remark 5 It is remarkable the level of generality of Theorem 2, which implies the existence of both $V @ R_{-} G P s$ and $V @ R$ GCs under quite weak assumptions. One is dealing with wealths $y \in L^{0}$ whose expectation does not have to be finite, the pricing rule $\Pi$ does not have to be linear, $\Pi$ does not have to be continuous, if $Y \subset L^{1}$ then risk aversion is not required ( $\Pi$ may be risk neutral or risk acceptant), etc.

Remark 6 Theorem 2 may apply in both, finance and insurance. An obvious implication in finance is that the most important arbitrage-free continuous time pricing models of Financial Mathematics (BS, stochastic volatility, etc.) allow us to find both $V @ R_{-} G P s$ and $V @ R \_G C s$. Furthermore, Theorem 2 easily shows that discrete time pricing models often lead to the existence of $V @ R \_G P s$ and $V @ R_{-} G P s$ as well. On the other hand, if $S_{T}$ represents total claims at $T$ for an insurer, and $\Pi$ is a reinsurer premium principle satisfying the required conditions above, ${ }^{6}$ then the sale of the stop-loss contract $\left(S_{T}-K_{\beta}\right)^{+}$with deductible $K_{\beta}$ may be very attractive for the reinsurer. This is important because the purchase of stop-loss contracts is often attractive for the direct insurer (Balbás et al., 2015).

Remark 7 ( $V @ R \_G P s$ and $V @ R \_G C s$ in the BS model). Theorem 2 and the remarks above show that Equations (8) and (9) always have solutions $k_{\beta}$ and $K_{\beta}$ under the classical assumptions of the BS model. Thus, let us give closed expressions for both strikes. Take $a=0$ and $b=\infty$. Suppose that $\Phi$ denotes the cumulative distribution function of a standard normal random variable. Consider the initial condition $S=S_{0}$ at $t=0$, as well as the $B S$

\footnotetext{
${ }^{6}$ the usual premium principles satisfy the required conditions (Pichler, 2014, Balbás et al., 2015, Cheung et al., 2015, etc.).
} 
stochastic differential equation $d S=S(\mu d t+\sigma d w), \mu \in \mathbb{R}$ being drift, $\sigma>0$ being volatility, and $w$ being a standard Brownian motion. It is known that

$$
\mathbb{P}(0, k)=\mathbb{P}\left(0<S_{T}<k\right)=\Phi\left(\frac{\log \left(k / S_{0}\right)-\left(\mu-\frac{\sigma^{2}}{2}\right) T}{\sigma \sqrt{T}}\right) .
$$

Therefore, (8) leads to

$$
k_{\beta}=S_{0} \operatorname{Exp}\left(\left(\mu-\frac{\sigma^{2}}{2}\right) T+\sigma \sqrt{T} \Phi^{-1}(1-\beta)\right)
$$

and

$$
\beta=\Phi\left(\frac{\left(\mu-\frac{\sigma^{2}}{2}\right) T-\log \left(k_{\beta} / S_{0}\right)}{\sigma \sqrt{T}}\right) .
$$

Similarly, (9) leads to

$$
K_{\beta}=S_{0} \operatorname{Exp}\left(\left(\mu-\frac{\sigma^{2}}{2}\right) T+\sigma \sqrt{T} \Phi^{-1}(\beta)\right)
$$

and

$$
\beta=\Phi\left(\frac{\log \left(K_{\beta} / S_{0}\right)-\left(\mu-\frac{\sigma^{2}}{2}\right) T}{\sigma \sqrt{T}}\right) .
$$

Besides, (5) and (6) show that the classical BS formula provides us with upper bounds for the $V @ R$ of the self-financing strategies of Theorem 2. The table below summarizes some numerical experiments implemented with the underlying asset current price $S_{0}=1000$, Expressions (12), (13), (14) and (15), and the above mentioned $V @ R$ upper bound.

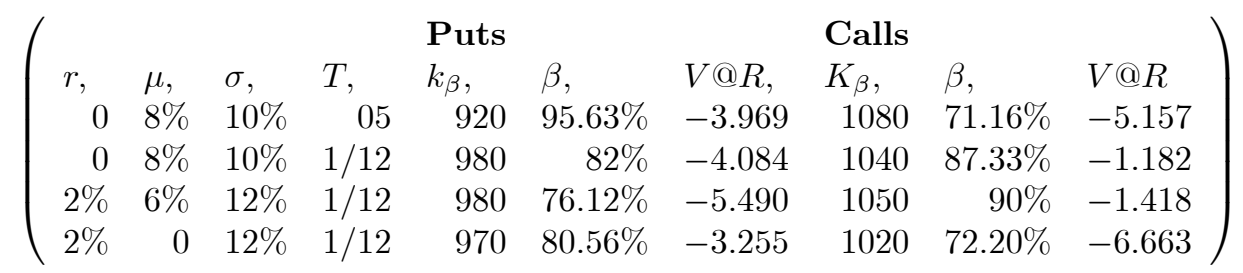

Notice that the $V @ R$ absolute value is always higher than $0.1 \%$ of the underlying asset price, and it is often higher than $0.3 \%$ of the same value, suggesting that both the $V @ R \_G P$ and the $V @ R \_G C$ may be very attractive to investors under the $B S$ framework. Furthermore, it is remarkable that the portfolio expected payoff at $T$ is always positive. For instance, for the $V @ R \_G P$ this pay-off becomes $1.3,0.2,0.05$, and 0.01 , respectively. Notice also that the sensitivity of the $V @ R$ bound with respect to the parameters $r, \mu, \sigma$ and $T$ may be easily computed by taking derivatives in (12), (13), (14), (15) and the classical BS formula. 


\section{Coherent risk measures and golden options}

With the notations of the proof of Theorem 2, and according to the $V @ R$ representation theorem of Balbás et al. (2017), if the strategy of Theorem $2 a$ has a finite expectation then its $C V @ R_{\beta}$ may be approximated by

$$
\lambda+\frac{1}{1-\beta} \mathbb{E}\left(\lambda_{M}\right)=-e^{r T} \Pi\left(\left(k_{\beta}-S_{T}\right)^{+}\right)+\frac{1}{1-\beta} \mathbb{E}\left(\left(k_{\beta}-S_{T}\right)^{+}\right) .
$$

Under the assumptions of the usual pricing models, $e^{r T} \Pi\left(\left(k_{\beta}-S_{T}\right)^{+}\right)$will equal the risk-neutral expectation of $\left(k_{\beta}-S_{T}\right)^{+}$and, consequently, though $\mathbb{E}\left(\left(k_{\beta}-S_{T}\right)^{+}\right)$is a physical (but not risk-neutral) expectation, for $\beta$ large enough the $C V @ R_{\beta}$ above will often become positive. The remainder sections will be devoted to studying whether one can also find golden options for the $C V @ R_{\beta}$ and other coherent risk measures.

Fix $1 \leq p<\infty$ and a risk measure $\rho: L^{p} \longrightarrow \mathbb{R}$ satisfying a representation theorem in the line of Artzner et al. (1999) and Rockafellar et al. (2006). More precisely, consider the conjugate $1<q \leq \infty$ of $p$ (i.e., $1 / p+1 / q=1$, with the convention $1 / \infty=0)$ and the sub-gradient of $\rho$

$$
\Delta_{\rho}:=\left\{z \in L^{q} ;-\mathbb{E}(y z) \leq \rho(y), \forall y \in L^{p}\right\} \subset L^{q}
$$

composed of those linear and continuous functions lower than $\rho$. We will assume that $\Delta_{\rho}$ is convex and weak $k^{*}$-compact (Kopp, 2008), and $\rho$ is its envelope, in the sense that

$$
\rho(y)=\operatorname{Max}\left\{-\mathbb{E}(y z) ; z \in \Delta_{\rho}\right\}
$$

holds for every $y \in L^{p}$. Furthermore, we will also assume that

$$
\{1\} \subset \Delta_{\rho} \subset\left\{z \in L^{q} ; \mathbb{E}(z)=1\right\}
$$

and

$$
\Delta_{\rho} \subset\left\{z \in L^{q} ; \mathbb{P}(z \geq 0)=1\right\} .
$$

Assumptions above are equivalent to the properties of norm-continuity, subadditivity, homogeneity, mean dominance, translation invariance and monotonicity. To sum up, we have:

Assumption $1 \rho: L^{p} \longrightarrow \mathbb{R}$ satisfies the equivalent conditions $\left.a\right)$ and $b$ ) below:

a) $\rho$ is norm-continuous, sub-additive $\left(\rho\left(y_{1}+y_{2}\right) \leq \rho\left(y_{1}\right)+\rho\left(y_{2}\right)\right.$ if $y_{1}, y_{2} \in$ $\left.L^{p}\right)$, homogeneous $\left(\rho(\varkappa y)=\varkappa \rho(y)\right.$ if $y \in L^{p}$ and $\left.\varkappa>0\right)$, mean dominating $\left(\rho(y) \geq-\mathbb{E}(y)\right.$ if $\left.y \in L^{p}\right)$, translation invariant $(\rho(y+\varkappa)=\rho(y)-\varkappa$ if $y \in L^{p}$ and $\left.\varkappa \in \mathbb{R}\right)$ and decreasing $\left(\rho\left(y_{1}\right) \leq \rho\left(y_{2}\right)\right.$ if $y_{1}, y_{2} \in L^{p}$ and $\left.\mathbb{P}\left(y_{1}-y_{2} \geq 0\right)=1\right)$.

b) The set (16) is convex and weak $k^{*}$ compact, (17) holds for every $y \in L^{p}$, and (18) and (19) hold. 
There are many examples of risk measures satisfying Assumption 1. A very famous one is the $C V @ R_{\beta}$, applying for every $p \in[1, \infty)$, and characterized by the sub-gradient

$$
\Delta_{C V @ R_{\beta}}=\left\{z \in L^{\infty} ; \mathbb{E}(z)=1,0 \leq z \leq 1 /(1-\beta)\right\} .
$$

With respect to the convex cone $Y \subset L^{0}$ and the pricing rule $\Pi$ of Section 2 above, we will assume that $Y=L^{p}$, $\Pi$ is continuous, ${ }^{7}$ and $r=R$. As a consequence of the Hahn-Banach Theorem (Schaeffer, 1970), the set

$$
\Delta_{\Pi}:=\left\{z \in L^{q} ; \mathbb{E}(y z) \leq e^{r T} \Pi(y), \forall y \in L^{p}\right\} \subset L^{q}
$$

is weak $^{*}$-compact and

$$
e^{r T} \Pi(y)=\operatorname{Max}\left\{\mathbb{E}(y z) ; z \in \Delta_{\Pi}\right\}
$$

holds for every $y \in L^{p}$. Moreover, (4) implies that

$$
\Delta_{\Pi} \subset\left\{z \in L^{q} ; \mathbb{E}(z)=1\right\}
$$

and (3) implies that

$$
\Delta_{\Pi} \subset\left\{z \in L^{q} ; \mathbb{P}(z \geq 0)=1\right\} .
$$

Remark 8 Expressions (17) and (21) imply that $\rho: L^{p} \longrightarrow \mathbb{R}$ and $\Pi: L^{p} \longrightarrow$ $\mathbb{R}$ are lower semi-continuous functions if $p>1$ and $L^{p}$ is endowed with the weak ${ }^{*}-$ topology.

If $\Pi$ is linear then $\Delta_{\Pi}=\left\{z_{\Pi}\right\}$ becomes a singleton, and it is known that $z_{\Pi}$ is called stochastic discount factor $(S D F)$ of $\Pi$ (Duffie, 1988). (21) leads to

$$
\Pi(y)=e^{-r T} \mathbb{E}\left(y z_{\Pi}\right)
$$

for every $y \in L^{p}$, and (22) leads to

$$
\mathbb{E}\left(z_{\Pi}\right)=1 .
$$

Remark 9 (4), Proposition $1 c$ and Assumption 1 obviously imply that

$$
\rho(y+\varkappa)+e^{r T} \Pi(y+\varkappa)=\rho(y)+e^{r T} \Pi(y)
$$

for every $y \in L^{p}$ and every $\varkappa \in \mathbb{R}$. Consider $y \in L^{p}$ such that

$$
\rho(y)+e^{r T} \Pi(y)<0 .
$$

If $\varkappa=-e^{r T} \Pi(y)$ then $y+\varkappa$ will be a self-financing strategy ((4) and Proposition $1)$ such that $\rho(y+\varkappa)<0$. Moreover, $\rho$ being mean dominating (Assumption $1)$, we have that $\mathbb{E}(y+\varkappa) \geq-\rho(y+\varkappa)>0$. In other words, the investor can

\footnotetext{
${ }^{7}$ many results still hold if $Y$ is a closed subspace of $L^{p}$, though we prefer to impose $Y=L^{p}$ in order to simplify the mathematical exposition.
} 
construct a self-financing portfolio with strictly negative risk and generating a strictly positive expected wealth. This is more than the situation we were facing when trading the $V @ R \_G P$ or the $V @ R \_G C$, but the role of $V @ R$ is now plaid by $\rho$. Furthermore, as in (10), we have that

$$
\left\{\begin{array}{l}
\Pi(n(y+\varkappa))=0, \\
\lim _{n} \rho(n(y+\varkappa))=-\infty \\
\lim _{n} \mathbb{E}(n(y+x))=+\infty
\end{array} \quad \forall n \in \mathbb{N}\right.
$$

i.e., if the investor borrows the price of $y$ and purchases $y$ as many times as desired, then he/she can construct strategies whose price always vanishes, whose risk becomes as negative as desired, and whose expected pay-off is positive and as high as desired.

\subsection{Smooth good deals}

Sequences satisfying (27) have been created in Balbás et al. (2016), among others, but every strategy in the sequence had to contain a digital option, whose replication may become complex in practice. Let us construct the sequence above with free of jumps derivatives.

We will assume that $S_{T}$ is a random variable with finite expectation and variance. Obviously, this property holds if and only if Assumption 2 below holds.

Assumption 2 Henceforth we will take $p=2$. Moreover, we will assume that he identity map $(a, b) \ni x \longrightarrow I(x)=x \in \mathbb{R}$ belongs to $L^{2}(\mathbb{P})$.

Fix $a_{0} \in[a, b)$ such that $a_{0} \neq-\infty$ and $\mathbb{P}\left(S_{T}=a_{0}\right)=0,{ }^{8}$ and for every $x \in L^{\infty}(\mathbb{L})$ and every $u \in(a, b)$ let us consider

$$
J(x)(u):=\int_{a_{0}}^{u} x(v) d v .
$$

Proposition $10 L^{\infty}(\mathbb{L}) \ni x \longrightarrow J(x) \in L^{2}(\mathbb{P})$ is a linear and continuous functional.

Proof. We have that

$$
J(x)(u)^{2}=\left|\int_{a_{0}}^{u} x(v) d v\right|^{2} \leq\left(\int_{a_{0}}^{u}\|x\|_{\infty} d v\right)^{2} \leq\|x\|_{\infty}^{2}\left(u-a_{0}\right)^{2} .
$$

Hence,

$$
\int_{a}^{b} J(x)(u)^{2} \mathbb{P}(d u) \leq\|x\|_{\infty}^{2} \int_{a}^{b}\left(u-a_{0}\right)^{2} \mathbb{P}(d u)=
$$

\footnotetext{
${ }^{8}$ we will usually take $a_{0}=a$, unless $a=-\infty$.
} 


$$
\|x\|_{\infty}^{2}\left(\int_{a}^{b} u^{2} \mathbb{P}(d u)-2 a_{0} \int_{a}^{b} u \mathbb{P}(d u)+a_{0}^{2} \int_{a}^{b} \mathbb{P}(d u)\right) .
$$

Obviously $\int_{a}^{b} u^{2} \mathbb{P}(d u)<\infty$ due to Assumption $2, \int_{a}^{b}|u| \mathbb{P}(d u)<\infty$ because the identity map is in $L^{1}(\mathbb{P})$ too (recall that $L^{2}(\mathbb{P}) \subset L^{1}(\mathbb{P})$ ), and $\int_{a}^{b} \mathbb{P}(d u)=1$. Therefore there exists a constant $\varkappa$ such that $\|J(x)\|_{2} \leq \varkappa\|x\|_{\infty}$, which implies that $J(x) \in L^{2}(\mathbb{P})$ and $J$ is continuous (Schaeffer, 1970).

Remark 11 In practice $x \in L^{\infty}(\mathbb{L})$ will be understood as the first order Greek (delta) at $T$ of the derivative security $J(x)$ we are interested in. For instance, if $(a, b)=(0, \infty), a_{0}=0$ and

$$
x= \begin{cases}0, & u<1 \\ 1, & u>1\end{cases}
$$

then

$$
J(x)(u)= \begin{cases}0, & u<1 \\ u-1, & u>1\end{cases}
$$

so $J(x)$ will be an European call option with strike 1 and maturity at $T$, and $x$ will be its delta at maturity

Besides, $J(x)$ is obviously continuous for every $x \in L^{\infty}(\mathbb{L})$ and, consequently, every $J(x)$ will be said to be a "smooth derivative of $S_{T}$ ".

If $\left(L^{\infty}(\mathbb{L})\right)^{\prime}$ represents the dual space of $L^{\infty}(\mathbb{L})$, then recall that $L^{1}(\mathbb{L}) \subset$ $\left(L^{\infty}(\mathbb{L})\right)^{\prime}$. Besides, one can consider the adjoint functional of $J$, denoted by $J^{*}: L^{2}(\mathbb{P}) \longrightarrow\left(L^{\infty}(\mathbb{L})\right)^{\prime}$. With the obvious notations for the classical bilinear products, $J^{*}$ is characterized by

$$
\langle J(x), y\rangle=\left\langle x, J^{*}(y)\right\rangle
$$

for every $x \in L^{\infty}(\mathbb{L})$ and every $y \in L^{2}(\mathbb{P})$ (Schaeffer, 1970).

Proposition $12 J^{*}: L^{2}(\mathbb{P}) \longrightarrow L^{1}(\mathbb{L})$ is linear and continuous, and it is given by

$$
J^{*}(y)(v)=\left\{\begin{array}{rll}
\int_{v}^{b} y(u) \mathbb{P}(d u) & \text { if } & v>a_{0} \\
-\int_{a}^{v} y(u) \mathbb{P}(d u) & \text { if } & v<a_{0}
\end{array}\right.
$$

for every $y \in L^{2}(\mathbb{P})$ and every $v \in(a, b)$.

Proof. It is a classical result that $J^{*}: L^{2}(\mathbb{P}) \longrightarrow\left(L^{\infty}(\mathbb{L})\right)^{\prime}$ is linear and continuous (Schaeffer, 1970). Thus, we only have to show that $J^{*}$ is valued in 
$L^{1}(\mathbb{L})$ and that $(30)$ holds. For $x \in L^{\infty}(\mathbb{L})$ and $y \in L^{2}(\mathbb{P})$ we have (see (29))

$$
\begin{aligned}
& \langle J(x), y\rangle=\int_{a}^{b} J(x)(u) y(u) \mathbb{P}(d u)= \\
& \int_{a}^{b}\left(\int_{a_{0}}^{u} x(v) d v\right) y(u) \mathbb{P}(d u)= \\
& \int_{a_{0}}^{b}\left(\int_{a_{0}}^{u} x(v) d v\right) y(u) \mathbb{P}(d u)-\int_{a}^{a_{0}}\left(\int_{u}^{a_{0}} x(v) d v\right) y(u) \mathbb{P}(d u)= \\
& \int_{a_{0}}^{b}\left(\int_{v}^{b} y(u) \mathbb{P}(d u)\right) x(v) d v-\int_{a}^{a_{0}}\left(\int_{a}^{v} y(u) \mathbb{P}(d u)\right) x(v) d v= \\
& \int_{a}^{b} J^{*}(y)(v) x(v) d v .
\end{aligned}
$$

Thus, (30) becomes obvious, and $J^{*}(y) \in L^{1}(\mathbb{L})$ because taking

$$
x=\left\{\begin{aligned}
1, & J^{*}(y) \geq 0 \\
-1, & J^{*}(y)<0
\end{aligned}\right.
$$

we have that $\int_{a}^{b}\left|J^{*}(y)(v)\right| d v=\int_{a}^{b} J^{*}(y)(v) x(v) d v=\langle J(x), y\rangle<\infty$.

As already said, our objective is to find a smooth derivative $y=J(x)$ satisfying (26). If such a $x$ does exist then it will be said to be a smooth good deal $(S G D)$ of $\left(L^{2}, \rho, \Pi\right) .^{9}$

Remark 13 One could attempt to compute the optimal SGD by solving the optimization problem

$$
\left\{\begin{array}{l}
\operatorname{Min} \rho(J(x))+e^{r T} \Pi(J(x)) \\
x \in L^{\infty}
\end{array}\right.
$$

However, it is obvious that (31) is bounded if and only if its optimal value equals 0 (recall that $J, \Pi$ and $\rho$ are positively homogeneous), in which case there is no $S G D$. A natural way to solve this caveat is to deal with problem

$$
\left\{\begin{array}{l}
\operatorname{Min} \rho(J(x))+e^{r T} \Pi(J(x)) \\
x \in L^{\infty},\|x\|_{\infty} \leq C
\end{array}\right.
$$

for some $C>0$. This is exactly the solution we propose, though we will consider $C=1$ because it is easy to see that the solution of (32) is given by the product of $C$ and the solution of (32) when $C=1$. Thus,

$$
\operatorname{Min} \rho(J(x))+e^{r T} \Pi(J(x))\left\{\begin{array}{l}
x \in L^{\infty} \\
-1 \leq x \leq 1
\end{array}\right.
$$

is the problem we will deal with.

\footnotetext{
${ }^{9}$ Good deals were introduced in Cochrane and Saa-Requejo (2000). Briefly speaking, they were strategies with a very high classical Sharpe ratio. In this paper we will interpret that a $G D$ is a strategy satisfying (26).
} 
Proposition $14 L^{\infty}(\mathbb{L}) \ni x \longrightarrow \rho(J(x))+e^{r T} \Pi(J(x)) \in \mathbb{R}$ is lower semicontinuous if $L^{\infty}(\mathbb{L})$ is endowed with the weak ${ }^{*}$-topology.

Proof. First, let us show that $L^{\infty}(\mathbb{L}) \ni x \longrightarrow J(x) \in L^{2}(\mathbb{P})$ is continuous if $L^{\infty}(\mathbb{L})$ and $L^{2}(\mathbb{P})$ are endowed with their weak $k^{*}$-topologies. Indeed, consider a net $\left(x_{i}\right)_{i \in I} \subset L^{\infty}(\mathbb{L})$ converging to zero in the weak ${ }^{*}$-topology of $L^{\infty}(\mathbb{L})$. For every $z \in L^{2}(\mathbb{P})$ we have that (see (29))

$$
\lim _{i \in I}\left\langle J\left(x_{i}\right), z\right\rangle=\lim _{i \in I}\left\langle x_{i}, J^{*}(z)\right\rangle=0
$$

where the last equality holds because $J^{*}(z) \in L^{1}(\mathbb{L})$ (Proposition 12) and $\left(x_{i}\right)_{i \in I}$ converges to zero in the weak ${ }^{*}$-topology of $L^{\infty}(\mathbb{L})$. (34) shows that $\left(J\left(x_{i}\right)\right)_{i \in I} \subset L^{2}(\mathbb{P})$ converges to zero in the weak - topology of $L^{2}(\mathbb{P})$, and $J$ is continuous for these topologies. Since $\rho$ is lower semi-continuos when the space $L^{2}(\mathbb{P})$ is endowed with the weak $k^{*}$-topology (Remark 8), we have that $\rho \circ J$ is also lower semi-continuous. Furthermore, a similar argument proves that $\Pi \circ J$ is weak $k^{*}$-lower semi-continuous too.

Proposition 15 Problem (33) is bounded and solvable.

Proof. The set $\left\{x \in L^{\infty}(\mathbb{L}) ;-1 \leq x \leq 1\right\}$ is weak ${ }^{*}$-compact (Alaoglu's theorem, Kopp, 2008), and therefore (33) is solvable because the objective function is lower semi-continuos (Proposition 14).

Proposition 16 There exists a SGD if and only if the optimal value of Problem (33) is strictly negative. Otherwise the optimal value of (33) equals zero.

Proof. Since $x=0$ is obviously (33)-feasible then the optimal value of (33) will never be positive. If it is zero then there will not exist any feasible $x$ such that $\rho(J(x))+e^{r T} \Pi(J(x))<0$, i.e., there will not exist any $S G D$. Conversely, if the optimal value is negative then $\rho(J(x))+e^{r T} \Pi(J(x))<0$ will hold for some (33)-feasible $x$.

\subsection{Dual approach}

This sub-section will be devoted to analyzing a Lagrangian dual problem for (33), which will provide us with new characterizations of the absence of $S G D$ as well as with new methods to compute the solution of (33).

Remark 17 (17) and (21) show that (33) is equivalent to the linear optimization problem

$$
\text { Min } \theta_{1}+\theta_{2} \begin{cases}-\mathbb{E}\left(J(x) z_{1}\right)-\theta_{1} \leq 0, & \forall z_{1} \in \Delta_{\rho} \\ \mathbb{E}\left(J(x) z_{2}\right)-\theta_{2} \leq 0, & \forall z_{2} \in \Delta_{\Pi} \\ -1 \leq x \leq 1 & \end{cases}
$$

$\left(\theta_{1}, \theta_{2}, x\right) \in \mathbb{R} \times \mathbb{R} \times L^{\infty}$ being the decision variable. The Lagrangian of (35) may be obtained with the general methodology of Balbás et al. (2015), and becomes

$$
\mathcal{L}\left(x, z_{1}, z_{2}, w_{1}, w_{2}\right)=\mathbb{E}\left(J(x) z_{2}\right)-\mathbb{E}\left(J(x) z_{1}\right)+\left\langle x-1, w_{1}\right\rangle-\left\langle x+1, w_{2}\right\rangle
$$


with $\left(x, z_{1}, z_{2}, w_{1}, w_{2}\right) \in L^{\infty} \times \Delta_{\rho} \times \Delta_{\Pi} \times\left(L^{\infty}(\mathbb{L})\right)^{\prime} \times\left(L^{\infty}(\mathbb{L})\right)^{\prime}, w_{1} \geq 0$ and $w_{2} \geq 0$. (29) leads to

$$
\mathcal{L}\left(x, z_{1}, z_{2}, w_{1}, w_{2}\right)=\left\langle x, J^{*}\left(z_{2}-z_{1}\right)+w_{1}-w_{2}\right\rangle-\left\langle 1, w_{1}+w_{2}\right\rangle .
$$

Following Luenberger (1969) and Balbás et al. (2015), $\left(z_{1}, z_{2}, w_{1}, w_{2}\right)$ is dualfeasible if and only if $\mathcal{L}\left(x, z_{1}, z_{2}, w_{1}, w_{2}\right)$ is bounded from below for $x \in L^{\infty}$, in which case its infimum equals the dual objective. Obviously, $\mathcal{L}\left(x, z_{1}, z_{2}, w_{1}, w_{2}\right)$ is bounded if and only if $\left\langle x, J^{*}\left(z_{2}-z_{1}\right)+w_{1}-w_{2}\right\rangle=0$ for every $x \in L^{\infty}$, in which case the dual problem will maximize $-\left\langle 1, w_{1}+w_{2}\right\rangle$. Consequently, one can take the dual problem

$$
\operatorname{Min}\left\langle 1, w_{1}+w_{2}\right\rangle\left\{\begin{array}{l}
J^{*}\left(z_{2}-z_{1}\right)+w_{1}-w_{2}=0 \\
w_{1} \geq 0, w_{2} \geq 0 \\
\left(z_{1}, z_{2}, w_{1}, w_{2}\right) \in \Delta_{\rho} \times \Delta_{\Pi} \times\left(L^{\infty}(\mathbb{L})\right)^{\prime} \times\left(L^{\infty}(\mathbf{L})\right)^{\prime}
\end{array}\right.
$$

$\left(z_{1}, z_{2}, w_{1}, w_{2}\right)$ being the decision variable. Since (35) is solvable (Proposition 15), the constraints of (35) are valued in spaces whose positive cone has non void interior (Schaeffer, 1974), and $\left(\theta_{1}, \theta_{2}, x\right)=(1,1,0)$ makes (35) satisfy the Slater qualification (Luenberger, 1969), there is no duality gap between (33) and (36) (the optimal value of (33) equals the optimal value of (36) with the opposite sign), and (36) is solvable (Luenberger, 1969). Moreover, there are Karush-Kuhn-Tucker like conditions characterizing the solutions of both (33) and (36). These conditions imply that the solutions $\tilde{x}$ and $\left(\tilde{z}_{1}, \tilde{z}_{2}, \tilde{w}_{1}, \tilde{w}_{2}\right)$ must satisfy the complementary slackness rules

$$
\left\{\begin{array}{l}
\left\langle\tilde{x}-1, \tilde{w}_{1}\right\rangle=0 \\
\left\langle\tilde{x}+1, \tilde{w}_{2}\right\rangle=0
\end{array}\right.
$$

Since $\tilde{x}-1=0$ and $\tilde{x}+1=0$ cannot simultaneously hold, we have that $\tilde{w}_{1}=0$ or $\tilde{w}_{2}=0$ for every state of nature. Furthermore, $J^{*}\left(\tilde{z}_{2}-\tilde{z}_{1}\right)+\tilde{w}_{1}-\tilde{w}_{2}=0$ and Proposition 12 show that $J^{*}\left(z_{2}-z_{1}\right) \in L^{1}(\mathbb{L})$, and the three conditions

$$
\left\{\begin{array}{l}
J^{*}\left(\tilde{z}_{2}-\tilde{z}_{1}\right)=-\tilde{w}_{1}+\tilde{w}_{2} \\
\tilde{w}_{1} \geq 0, \tilde{w}_{2} \geq 0, \tilde{w}_{1}=0 \text { or } \tilde{w}_{2}=0 \\
J^{*}\left(\tilde{z}_{2}-\tilde{z}_{1}\right) \in L^{1}(\mathbf{L L})
\end{array}\right.
$$

imply that $J^{*}\left(\tilde{z}_{2}-\tilde{z}_{1}\right)=-\tilde{w}_{1}$ for some states of nature and $J^{*}\left(\tilde{z}_{2}-\tilde{z}_{1}\right)=\tilde{w}_{2}$ for the rest of states. In other words, $\left(\tilde{w}_{1}, \tilde{w}_{2}\right) \in L^{1}(\mathbb{L}) \times L^{1}(\mathbb{L})$. To sum up, if one denotes $w^{+}=w_{1}$ and $w^{-}=w_{2}$, and one bears in mind that

$$
\left\langle 1, w^{+}+w^{-}\right\rangle=\int_{a}^{b}\left(w^{+}+w^{-}\right) d \mathbb{L}
$$

then one has (see Balbás et al., 2015):

Theorem 18 Problem

$$
\operatorname{Min} \int_{a}^{b}\left(w^{+}+w^{-}\right) d \mathbf{L}\left\{\begin{array}{l}
J^{*}\left(z_{1}\right)-w^{+}+w^{-}=J^{*}\left(z_{2}\right) \\
w^{+} \geq 0, w^{-} \geq 0 \\
\left(z_{1}, z_{2}, w^{+}, w^{-}\right) \in \Delta_{\rho} \times \Delta_{\Pi} \times L^{1}(\mathbf{I L}) \times L^{1}(\mathbf{L L})
\end{array}\right.
$$


is the dual of (33), it is bounded and solvable, and its optimal value equals the optimal value of (33) multiplied by -1 . Moreover, if $\tilde{x}$ and $\left(\tilde{z}_{1}, \tilde{z}_{2}, \tilde{w}^{+}, \tilde{w}^{-}\right)$are (33) and (37)-feasible respectively, then they solve the corresponding optimization problem if and only if the following Karush-Kuhn-Tucker like conditions

$$
\begin{cases}\mathbb{E}(J(\tilde{x}) z) \geq \mathbb{E}\left(J(\tilde{x}) \tilde{z}_{1}\right), & \forall z \in \Delta_{\rho} \\ \mathbb{E}(J(\tilde{x}) z) \leq \mathbb{E}\left(J(\tilde{x}) \tilde{z}_{2}\right), & \forall z \in \Delta_{\Pi} \\ \left\langle\tilde{x}-1, \tilde{w}^{+}\right\rangle=\left\langle\tilde{x}+1, \tilde{w}^{-}\right\rangle=0 & \end{cases}
$$

hold.

Corollary 19 There is no $S G D$ if and only if there exist $\tilde{z}_{1} \in \Delta_{\rho}$ and $\tilde{z}_{2} \in \Delta_{\Pi}$ such that $J^{*}\left(\tilde{z}_{1}\right)=J^{*}\left(\tilde{z}_{2}\right)$. If so, $\left(\tilde{z}_{1}, \tilde{z}_{2}, \tilde{w}^{+}, \tilde{w}^{-}\right)=\left(\tilde{z}_{1}, \tilde{z}_{2}, 0,0\right)$ solves (37).

Proof. Proposition 16 and Theorem 18 show that there is no $S G D$ if and only if the optimal value of (37) equals zero. The constraints of (37) imply that its objective vanishes if and only if $w^{+}=w^{-}=0$ are feasible for some $\left(\tilde{z}_{1}, \tilde{z}_{2}\right) \in \Delta_{\rho} \times \Delta_{\Pi}$, which is equivalent to $J^{*}\left(\tilde{z}_{1}\right)=J^{*}\left(\tilde{z}_{2}\right)$.

Remark 20 Readers with some knowledge about Goal Programming will realize that (37) is a linear goal programming problem, easy to solve in practice (see Anderson and Nash, 1987, Tamiz et al., 1998, Salas-Molina, 2018, etc.). Once (37) has been solved, the constraints of (33) and Conditions (38) will enable us to solve (33) and find the optimal SGD. Then, sequences satisfying (27) will be easy to create (see Remark 9).

(37) being a goal programming problem implies that its objective function equals $\left\|J^{*}\left(z_{1}\right)-J^{*}\left(z_{2}\right)\right\|_{1}$ (recall that $J^{*}\left(z_{1}\right), J^{*}\left(z_{2}\right) \in L^{1}(\mathbb{L})$, Proposition 12). Thus (37) may replaced by

$$
\left\{\begin{array}{l}
\operatorname{Min}\left\|J^{*}\left(z_{1}\right)-J^{*}\left(z_{2}\right)\right\|_{1} \\
\left(z_{1}, z_{2}\right) \in \Delta_{\rho} \times \Delta_{\Pi}
\end{array}\right.
$$

If $\left(\tilde{z}_{1}, \tilde{z}_{2}\right)$ solves (39) then the solution of (37) comes from, ${ }^{10}$

$$
\tilde{w}^{+}=\left(J^{*}\left(\tilde{z}_{1}\right)-J^{*}\left(z_{2}\right)\right)^{+} \text {and } \tilde{w}^{-}=\left(J^{*}\left(\tilde{z}_{1}\right)-J^{*}\left(z_{2}\right)\right)^{-}
$$

If $\Pi$ is linear (see (23)) and there is no SGD then the dual solution

$$
\left(\tilde{z}_{1}, \tilde{z}_{2}, \tilde{w}^{+}, \tilde{w}^{-}\right)=\left(\tilde{z}_{1}, z_{\Pi}, 0,0\right)
$$

will satisfy $J^{*}\left(\tilde{z}_{1}\right)=J^{*}\left(z_{\Pi}\right)$ and (see (23), and (29))

$$
\begin{aligned}
& \Pi(J(x))=e^{-r T} \mathbb{E}\left(J(x) z_{\Pi}\right)=e^{-r T}\left\langle x, J^{*}\left(z_{\Pi}\right)\right\rangle= \\
& e^{-r T}\left\langle x, J^{*}\left(\tilde{z}_{1}\right)\right\rangle=e^{-r T}\left\langle J(x), \tilde{z}_{1}\right\rangle=e^{-r T} \mathbb{E}\left(J\left(x_{1}\right) \tilde{z}\right),
\end{aligned}
$$

so $\tilde{z}_{1}$ may play the role of a SDF if one prices marketed claims of $J\left(L^{\infty}\right)$.

\footnotetext{
10 as usual, $h^{+}=\operatorname{Max}\{h, 0\}$ and $h^{-}=\operatorname{Max}\{-h, 0\}$ for every $h \in \mathbb{R}$.
} 


\subsection{Examples of golden option}

Consider $-\infty<a<b \leq \infty$ and suppose that $\mathbb{P}$ has a strictly positive RadonNikodym derivative with respect to $\mathbb{L}$ in $(a, b)$, and therefore $\mathbb{P}$ is atomless and its support equals $(a, b)$. Take $a_{0}=a$. Suppose that $\Pi$ is linear, and consider the $S D F z_{\Pi}$. Expressions (3) and (23) imply that

$$
\mathbb{P}\left(z_{\Pi}>0\right)=1 .
$$

(24), (30) and (41) lead to

$$
J^{*}\left(z_{\Pi}\right)(v)=\int_{v}^{b} z_{\Pi}(u) \mathbb{P}(d u)=\int_{v}^{b} \mathbb{Q}(d u)=\mathbb{Q}\left(S_{T} \geq v\right)
$$

for every $v \in(a, b), \mathbb{Q}$ being the risk-neutral probability of the pricing rule, ${ }^{11}$ which is equivalent to $\mathbb{P}$. In particular, $\mathbb{Q}$ is atomless with support $(a, b)$.

Theorem 21 Suppose that $\rho=C V @ R_{\beta}$. Suppose that $(a, b) \ni u \longrightarrow z_{\Pi}(u) \in$ $\mathbb{R}$ is one to one, strictly decreasing and continuous. The model is not $S G D$ free if and only if

$$
\lim _{u \rightarrow a} z_{\Pi}(u)>1 /(1-\beta) .
$$

If so, there exists $k_{\beta} \in(a, b)$ such that

$$
\tilde{x}=\chi_{\left(a, k_{\beta}\right)}= \begin{cases}1, & u<k_{\beta} \\ 0, & u>k_{\beta}\end{cases}
$$

solves (33) and

$$
\left\{\begin{array}{l}
\tilde{z}_{1}=\tilde{z}= \begin{cases}1 /(1-\beta), & u<k_{\beta} \\
z_{\Pi}, & u>k_{\beta}\end{cases} \\
\tilde{z}_{2}=z_{\Pi} \\
\tilde{w}^{+}(v)=J^{*}\left(\tilde{z}_{1}\right)(v)-\mathbb{Q}\left(S_{T} \geq v\right) \\
\tilde{w}^{-}=0
\end{array}\right.
$$

solves (37).

Proof. If $\lim _{u \rightarrow a} z_{\Pi}(u) \leq 1 /(1-\beta)$ then $z_{\Pi} \leq 1 /(1-\beta)$, and (24), (20) and (41) show that $z_{\Pi} \in \Delta_{C V a R_{\beta}}$. Corollary 19 shows that there is no $S G D$. Conversely, if there is no $S G D$ there exists $z \in \Delta_{C V a R_{\beta}}$ such that $J^{*}(z)=J^{*}\left(z_{\Pi}\right)$, i.e. (see $(30))$

$$
\int_{u}^{b} z(v) \mathbb{P}(d v)=\int_{u}^{b} z_{\Pi}(v) \mathbb{P}(d v)
$$

for every $u \in(a, b)$. Computing derivatives with respect to $u$, and bearing in mind that the Radon-Nikodym derivative of $\mathbb{P}$ with respect to $\mathbb{L}$ is strictly positive, we have that $z=z_{\Pi}$ out of a $\mathbb{P}$-null set, and consequently $z_{\Pi} \leq$ $1 /(1-\beta)$ due to $(20)$.

\footnotetext{
${ }^{11}$ i.e., the probability whose Radon-Nikodym derivative with respect to $\mathbb{P}$ equals $z_{\Pi}$.
} 
Suppose now that (43) holds. (24) and (43) imply that $\mathbb{P}\left(z_{\Pi}<1\right)>0$, and therefore $z_{\Pi}(u)<1<1 /(1-\beta)$ if $u$ is large enough. Hence, (43) and the Bolzano's Theorem imply that

$$
z_{\Pi}\left(k_{0}\right)=1 /(1-\beta)
$$

for some $k_{0} \in(a, b)$. Obviously, if

$$
z_{k}= \begin{cases}1 /(1-\beta), & u<k \\ z_{\Pi}, & u>k\end{cases}
$$

for every $k \in(a, b)$ one has that (see (30) and (42))

$$
\left\{\begin{array}{l}
\mathbb{E}\left(z_{k}\right)= \\
\int_{a}^{b} z_{k}(u) \mathbb{P}(d u)=\frac{\mathbf{P}\left(S_{T}<k\right)}{1-\beta}+J^{*}\left(z_{\Pi}\right)(k)=\frac{\mathbf{P}\left(S_{T}<k\right)}{1-\beta}+\mathbb{Q}\left(S_{T} \geq k\right) .
\end{array}\right.
$$

In particular,

$$
\lim _{k \rightarrow b} \mathbb{E}\left(z_{k}\right)=\frac{1}{1-\beta} \lim _{k \rightarrow b} \mathbb{P}\left(S_{T}<k\right)+\lim _{k \rightarrow b} \mathbb{Q}\left(S_{T} \geq k\right)=\frac{1}{1-\beta}>1 .
$$

For $k=k_{0}$ we have that (46) and (47) imply that $z_{k_{0}} \leq z_{\Pi}$ and $z_{k_{0}}(u)<z_{\Pi}(u)$ if $u<k_{0}$ ( $z_{\Pi}$ is strictly decreasing). Thus, (24) leads to

$$
\mathbb{E}\left(z_{k_{0}}\right)<\mathbb{E}\left(z_{\Pi}\right)=1 .
$$

(48) shows that $k \longrightarrow \mathbb{E}\left(z_{k}\right)$ is continuous ( $\mathbb{P}$ and $\mathbb{Q}$ are atomless), so the Bolzano's Theorem implies the existence of $k_{1} \in(a, b), k_{1}>k_{0}$, such that

$$
\mathbb{E}\left(z_{k_{1}}\right)=1 \text {. }
$$

Take $k_{\beta}=k_{1}$, and $\tilde{x}$ and $\left(\tilde{z}, z_{\Pi}, \tilde{w}^{+}, \tilde{w}^{-}\right)$as given by (44) and (45), respectively. $\tilde{x}$ is obviously (33)-feasible. (16), (41) and (49) show that $\tilde{z} \in \Delta_{C V a R_{\beta}}$ will hold if $\tilde{z}=z_{k_{\beta}} \leq 1 /(1-\beta)$. The inequality is obvious if $u<k_{\beta}$, and for $u>k_{\beta}=k_{1}>k_{0}$ one has $\tilde{z}(u)=z_{\Pi}(u)<z_{\Pi}\left(k_{0}\right)=1 /(1-\beta)$ (see (46)). Hence, $\tilde{z} \in \Delta_{C V a R_{\beta}}$. In order to show that $\left(\tilde{z}, z_{\Pi}, \tilde{w}^{+}, \tilde{w}^{-}\right)$is (37)-feasible one has to prove that

$$
J^{*}(\tilde{z})(v)-\tilde{w}^{+}=J^{*}\left(z_{\Pi}\right)(v) \quad \text { and } \quad \tilde{w}^{+} \geq 0 .
$$

The first condition trivially follows from (42) and (45). The second one trivially holds if $u \geq k_{\beta}$, since $z_{\Pi}(v)=\tilde{z}(v)$ for $v>k_{\beta}$ implies that $J^{*}\left(z_{\Pi}\right)(u)=$ $J^{*}(\tilde{z})(u)$ and $\tilde{w}^{+}(u)=J^{*}(\tilde{z})(u)-J^{*}\left(z_{\Pi}\right)(u)=0$. For $u<k_{\beta}$ we must prove that

$$
J^{*}(\tilde{z})(u)-J^{*}\left(z_{\Pi}\right)(u) \geq 0 .
$$

For $k_{0} \leq u<k_{\beta}$ one has

$$
J^{*}(\tilde{z})(u)-J^{*}\left(z_{\Pi}\right)(u)=\int_{u}^{k_{\beta}}\left(\frac{1}{1-\beta}-z_{\Pi}(v)\right) \mathbb{P}(d v)>0
$$


because $\frac{1}{1-\beta}-z_{\Pi}(v)>0$ (see (46) and recall that $z_{\Pi}$ is strictly decreasing). For $u<k_{0}(50)$ still applies, so $J^{*}(\tilde{z})(u)-J^{*}\left(z_{\Pi}\right)(u)$ is increasing because $\frac{1}{1-\beta}-z_{\Pi}(v)<0$. Therefore,

$$
J^{*}(\tilde{z})(u)-J^{*}\left(z_{\Pi}\right)(u) \geq \lim _{v \rightarrow a}\left(J^{*}(\tilde{z})(v)-J^{*}\left(z_{\Pi}\right)(v)\right)=\mathbb{E}(\tilde{z})-\mathbb{E}\left(z_{\Pi}\right)=0
$$

due to (18) and (24). Once we know that $\tilde{x}$ and $\left(\tilde{z}, z_{\Pi}, \tilde{w}^{+}, \tilde{w}^{-}\right)$are feasible Theorem 18 applies and it only remains to prove that they satisfy (38). The second and fourth conditions in (38) are trivial, so let us focus on the third one. If $\tilde{w}^{+}(u)>0$ then (45) leads to $J^{*}(\tilde{z})(u)>J^{*}\left(z_{\Pi}\right)(u)$, and the expression for $\tilde{z}$ implies that $u<k_{\beta}$. Therefore, (44) leads to $\tilde{x}=1$. Lastly, let us focus on the first condition of (38). Obviously,

$$
J(\tilde{x})= \begin{cases}u-a, & u<k_{\beta} \\ k_{\beta}-a, & u>k_{\beta}\end{cases}
$$

and

$$
\mathbb{E}(J(\tilde{x}) z)=\int_{a}^{b} J(\tilde{x}) z d \mathbb{P}
$$

and we must show that this functional is minimized by $\tilde{z}$ under the constraints of $\Delta_{C V a R_{\beta \alpha}}$, i.e.,

$$
\left\{\begin{array}{l}
\int_{a}^{b} z d \mathbb{P}=1 \\
0 \leq z \leq 1 /(1-\beta)
\end{array}\right.
$$

Sufficient optimality conditions for this linear problem are (Anderson and Nash, 1987)

$$
\left\{\begin{array}{l}
J(\tilde{x})-\lambda-\lambda_{1}+\lambda_{2}=0 \\
\lambda_{1} z=0 \\
\lambda_{2}\left(\frac{1}{1-\beta}-z\right)=0 \\
\lambda \in \mathbb{R}, \lambda_{1}, \lambda_{2} \in L^{2}(\mathbb{P}), \lambda_{1}, \lambda_{2} \geq 0
\end{array}\right.
$$

and (45) and (51) imply that $\tilde{z}$ trivially satisfies these conditions if

$$
\lambda_{2}= \begin{cases}k_{\beta}-u, & u<k_{\beta} \\ 0, & u>k_{\beta}\end{cases}
$$

$\lambda=k_{\beta}-a$ and $\lambda_{1}=0$.

Remark 22 (The $C V @ R$ golden put, $C V @ R \_G P$ ). Suppose that (43) holds. The Theorem's proof is constructive with respect to $k_{\beta}$, which will solve the equation

$$
\frac{\mathbb{P}\left(S_{T}<k\right)}{1-\beta}+\mathbb{Q}\left(S_{T}>k\right)=1
$$

or, equivalently,

$$
\mathbb{P}\left(S_{T} \leq k_{\beta}\right)=(1-\beta) \mathbb{Q}\left(S_{T} \leq k_{\beta}\right)
$$


Once $k_{\beta}$ has been estimated, the computation of $\tilde{x}$ and $\tilde{z}$ is a trivial application of (44) and (45). The marketed claim $J(\tilde{x})$ of (51) is the SGD we are looking for. This pay-off is easy to replicate by trading the riskless asset and selling the European put $\left(k_{\beta}-S_{T}\right)^{+}$, which will be called $C V @ R$ golden put $\left(C V @ R \_G P\right)$. Bearing in mind (25) and Remark 9, The sale of the $C V @ R_{-} G P$ an the investment of its price in the riskless asset is a self-financing strategy whose $C V @ R_{\beta}$ is negative and equals the optimal value of (33). Furthermore, if the strategy may be implemented several times, then (27) holds.

Remark 23 For the BS model, the Heston model, and most of the classical continuous-time stochastic volatility models, the assumptions of Theorem 21 and (43) hold if the model drift is higher than the riskless rate $(\mu>r)$, which is the usual situation. Moreover, $a=0$, and the limit in (43) equals $\infty$. Thus, all of these models present $C V @ R_{-} G P s$ for every level of confidence. If $\mu=$ $r$ then it is easy to prove that the model is $S G D$ free $\left(\mathbb{P}\left(z_{\Pi}=1\right)=1\right.$ and therefore (43) fails). If $\mu<r$ then these models are not $S G D$ free either, and a result quite parallel to Theorem 21 may be proved. Actually, the SDF becomes strictly increasing and the optimal $S G D$ becomes a $C V @ R$ golden European call $\left(C V @ R_{-} G C\right)$ whose strike $K_{\beta}$ solves the equation

$$
\mathbb{P}\left(S_{T} \geq k_{\beta}\right)=(1-\beta) \mathbb{Q}\left(S_{T} \geq k_{\beta}\right) .
$$

We will omit the proof due to its analogy with that of Theorem 21.

Remark 24 ( $C V @ R_{-} G P$ in the BS model). Consider the BS model and suppose that $\mu>r$. (11) and (52) lead to

$$
\Phi\left(\frac{\log \left(k_{\beta} / S_{0}\right)-\left(\mu-\frac{\sigma^{2}}{2}\right) T}{\sigma \sqrt{T}}\right)=(1-\beta) \Phi\left(\frac{\log \left(k_{\beta} / S_{0}\right)-\left(r-\frac{\sigma^{2}}{2}\right) T}{\sigma \sqrt{T}}\right) .
$$

The effect on $k_{\beta}$ of small changes in the input-side may be easily estimated by computing implicit differentials on Expression (53).

We have numerically solved Equation (53), and some results are reported in the table below. The columns "Exp" and " $C V @ R$ " give expectation and $C V @ R$ for a self-financing portfolio, i.e., the sale of the $C V @ R \_G P$ plus the investment in the riskless asset. We have assumed that $S_{0}=1000$.

$$
\left(\begin{array}{rllrlllr}
r, & \mu, & \sigma, & \multicolumn{1}{l}{T,} & \beta, & k_{\beta}, & \text { Exp, } & C V @ R \\
0 & 7 \% & 10 \% & 0.1 & 62 \% & 880 & 0.0002 & -0.00017 \\
0 & 7 \% & 10 \% & 0.5 & 60 \% & 920 & 1.07 & -0.60 \\
0 & 7 \% & 12 \% & 1 & 65 \% & 876 & 2.53 & -1.46 \\
1 \% & 8 \% & 10 \% & 0.1 & 70 \% & 849 & 1.75 & -5.00 \\
1 \% & 8 \% & 10 \% & 1 & 65 \% & 945 & 5.04 & -2.67 \\
1 \% & 8 \% & 9 \% & 0.1 & 75 \% & 860 & 9.13 & -2.23 \\
1 \% & 8 \% & 9 \% & 0.1 & 85 \% & 810 & 9.59 & -1.66 \\
1 \% & 8 \% & 9 \% & 1 & 80 \% & 898 & 2.03 & -0.70 \\
1 \% & 8 \% & 9 \% & 1 & 70 \% & 950 & 5.20 & -2.70
\end{array}\right)
$$


The numerical results are quite similar to those reported in Remark 7 for $V @ R$, though the level of confidence must be reduced. Actually, if the level of confidence and/or the volatility increase then it seems that the strategy expected pay-off decreases and the $C V @ R$ increases. Besides, these numerical results are consistent with the reports of the empirical papers by Bondarenko (2014) and Balbás et al. (2016). On the one hand, Bondarenko (2014) pointed out that the real quotation of OTM-puts is frequently higher than the price predicted by the classical pricing models. Needless to say, this is "good" for the CV@R_GP seller, who will improve both expected wealth and $C V @ R$ if he/she sells for prices bigger than those used in the table above. On the other hand, Balbás et al. (2016) constructed (non smooth) GDs under the assumptions of the BS model and under real market quotations, and they pointed out that the practical performance of a GD is much better if real quotes are used.

\section{Discrete models}

Many pricing models in Mathematical Finance are discrete, and they only involve finitely many trading dates and states of nature. Furthermore, most of the continuous pricing models may be accurately approximated by a discrete one. Thus, let us give a general practical procedure leading to the solutions of both (33) and (37) in general discrete models. This procedure will not require the additional assumptions of Theorem 21, and therefore it will enable us to give good approximations for $\tilde{x}$ and $\left(\tilde{z}_{1}, \tilde{z}_{2}, \tilde{w}^{+}, \tilde{w}^{-}\right)$in a very general framework. In order to shorten the exposition, we will assume that $\Pi$ is linear, though the extension to more general cases is not at all complex.

Suppose that the final underlying asset price $S_{T}$ will achieve a value belonging to the set $U=\left\{u_{0}<u_{1}<\ldots<u_{m}\right\} \subset \mathbb{R}$. Take $-\infty<a=a_{0}<u_{0}$ and $\infty>b>u_{m}$. Suppose that $U$ equals the support of $\mathbb{P}$. With the natural notation, if $y=\left(y\left(u_{0}\right), y\left(u_{1}\right), \ldots, y\left(u_{m}\right)\right)=\left(y_{0}, y_{1}, \ldots, y_{m}\right) \in L^{2}(\mathbb{P})$, then $(30)$ leads to

$$
J^{*}(y)(v)=\sum_{u_{j} \geq v} y\left(u_{j}\right) \mathbb{P}\left(S_{T}=u_{j}\right)
$$

for every $v \in(a, b)$. Thus,

$$
J^{*}(y)(v)=\sum_{j=h}^{m} \mathbb{P}\left(S_{T}=u_{j}\right) y_{j}
$$

for $v \in\left(u_{h-1}, u_{h}\right], h=0,1, \ldots m$ (take $\left.u_{-1}=a=a_{0}\right)$, and

$$
J^{*}(y)(v)=0
$$

for $v \in\left(u_{m}, b\right)$. In particular, if $y=z \in \Delta_{\rho}$ or $y=z_{\Pi}$ then (18) and (24) lead to

$$
J^{*}(y)(v)=1
$$

for every $v \in\left(a, u_{0}\right]$. 
With respect to the dual variables $w^{+}$and $w^{-}$of (37), under optimality they are given by (40). Thus, (40), (54), (55), (56) and the constraints of (37) show that the optimal solutions $\tilde{w}^{+}$and $\tilde{w}^{-}$(whose existence is guaranteed by Theorem 18) remain constant in $\left(u_{h-1}, u_{h}\right], h=0,1, \ldots, m$, and $\left(u_{m}, b\right)$. Moreover (55) and (56) show that $\tilde{w}^{+}$and $\tilde{w}^{-}$must vanish on $\left(a_{0}, u_{0}\right]$ and $\left(u_{m}, b\right)$. In other words, the dual variables $w^{+}$and $w^{-}$may simplify to $\left(w_{1}^{+}, \ldots, w_{m}^{+}\right)$ and $\left(w_{1}^{-}, \ldots, w_{m}^{-}\right)$, where every $w_{k}^{+}\left(w_{k}^{-}\right)$represents the value of $w^{+}\left(w^{-}\right)$on $\left(u_{h-1}, u_{h}\right], h=1,2, \ldots, m$. Hence, Problem (37) becomes

$$
\begin{cases}\operatorname{Min} \sum_{j=1}^{m}\left(u_{j}-u_{j-1}\right)\left(w_{j}^{+}+w_{j}^{-}\right) & \\ J^{*}(z)\left(u_{j}\right)-w_{j}^{+}+w_{j}^{-}=J^{*}\left(z_{\Pi}\right)\left(u_{j}\right), & j=1,2, \ldots, m \\ w_{j}^{+} \geq 0, w_{j}^{-} \geq 0, & j=1,2, \ldots, m \\ z=\left(z_{0}, z_{1}, \ldots, z_{m}\right) \in \Delta_{\rho} & \end{cases}
$$

$\left(z_{0}, z_{1}, \ldots, z_{m}, w_{1}^{+}, w_{2}^{+}, \ldots, w_{m}^{+}, w_{1}^{-}, w_{2}^{-}, \ldots, w_{m}^{-}\right) \in \mathbb{R}^{3 m-2}$ being the decision variable. Bearing in mind (54), (55) and (56), and denoting $z_{\Pi}=\left(z_{\Pi, 0}, z_{\Pi, 1}, \ldots, z_{\Pi . m}\right)$, the dual above becomes

$$
\begin{cases}\operatorname{Min} \sum_{j=1}^{m}\left(u_{j}-u_{j-1}\right)\left(w_{j}^{+}+w_{j}^{-}\right) & \\ \sum_{h=j}^{m} \mathbb{P}\left(S_{T}=u_{h}\right) z_{h}-w_{j}^{+}+w_{j}^{-}=\sum_{h=j}^{m} \mathbb{P}\left(S_{T}=u_{h}\right) z_{\Pi, h}, & j=1,2, \ldots, m \\ w_{j}^{+} \geq 0, w_{j}^{-} \geq 0, & j=1,2, \ldots, m \\ z=\left(z_{0}, z_{1}, \ldots, z_{m}\right) \in \Delta_{\rho} & \end{cases}
$$

Moreover, if (41) holds then the right hand side of the first constraint satisfies

$$
\sum_{h=j}^{m} \mathbb{P}\left(S_{T}=u_{h}\right) z_{\Pi, h}=\mathbb{Q}\left(S_{T} \geq u_{j}\right),
$$

$j=0,1,2, \ldots, m$, where, once again, $\mathbb{Q}$ is the risk-neutral probability, which is equivalent to $\mathbb{P}$. 
For $\rho=C V @ R_{\beta}$ one has (see (20))

$$
\begin{cases}\operatorname{Min} \sum_{j=1}^{m}\left(u_{j}-u_{j-1}\right)\left(w_{j}^{+}+w_{j}^{-}\right) & \\ \sum_{h=j}^{m} \mathbb{P}\left(S_{T}=u_{h}\right) z_{h}-w_{j}^{+}+w_{j}^{-}=\sum_{k=j}^{m} \mathbb{P}\left(S_{T}=u_{h}\right) z_{\Pi, h}, & j=1,2, \ldots, m \\ \sum_{j=1}^{m} \mathbb{P}\left(S_{T}=u_{j}\right) z_{j}=1 & \\ 0 \leq z_{j} \leq 1 /(1-\beta) & j=0,1, \ldots, m \\ w_{j}^{+} \geq 0, w_{j}^{-} \geq 0, & j=1,2, \ldots, m\end{cases}
$$

The goal programming problems (57) and (59) attain their optimal values (Theorem 18), and it is easy to solve them in practice (Tamiz et al., 1998).

Once a dual solution $\left(\tilde{z}_{0}, \ldots, \tilde{z}_{m}, \tilde{w}_{1}^{+}, \ldots, \tilde{w}_{m}^{+}, \tilde{w}_{1}^{-}, \ldots, \tilde{w}_{m}^{-}\right)$has been obtained, let us show that the optimal $\tilde{x}$ is easy to find as well. ${ }^{12}$ First of all, the value of $\tilde{x}(u)$ for $u \in\left(u_{m}, b\right)$ is irrelevant, because

$$
\mathbb{E}(J(\tilde{x}) z)=\sum_{j=0}^{m} z_{j} J(\tilde{x})\left(u_{j}\right) \mathbb{P}\left(S_{T}=u_{j}\right)
$$

for every $z \in L^{2}(\mathbb{P})$, and consequently (17) and (23) imply that only $J(\tilde{x})\left(u_{j}\right)$, $j=0,1, \ldots, m$, affect the objective function of (33). Obviously, (28) imply that $\tilde{x}(u)$ for $u \in\left(u_{m}, b\right)$ does not affect $J(\tilde{x})\left(u_{j}\right), j=0,1, \ldots, m$. Besides, one can suppose that $\tilde{x}$ remains constant on every $\left(u_{j-1}, u_{j}\right], j=0,1, \ldots, m$ (recall that $\left.u_{-1}=a=a_{0}\right)$. Indeed,

$$
\int_{u_{j-1}}^{u_{j}} \tilde{x}(v) d v=\frac{I}{u_{j}-u_{j-1}} \int_{u_{j-1}}^{u_{j}} d v
$$

where

$$
I=\int_{u_{j-1}}^{u_{j}} \tilde{x}(v) d v
$$

so $\tilde{x}$ can be replaced by $I /\left(u_{j}-u_{j-1}\right)$ in the involved interval. Clearly, $-1 \leq$ $\tilde{x} \leq 1$ implies that $-1 \leq I /\left(u_{j}-u_{j-1}\right) \leq 1$, and (60) implies that $J(\tilde{x})$ will equal $J\left(\tilde{x}^{\prime}\right)$ out of a $\mathbb{P}-$ null set if $\tilde{x}^{\prime}$ is the obvious piecewise function. Lastly, if $\tilde{x}(u)=x_{0}$ for $u \in\left(a_{0}, u_{0}\right]$ then take

$$
\tilde{x}^{\prime}= \begin{cases}0, & u \leq u_{0} \\ \tilde{x}, & \text { otherwise }\end{cases}
$$

and we will have

$$
J\left(\tilde{x}^{\prime}\right)(u)=\int_{a_{0}}^{u} \tilde{x}^{\prime} d v=J(\tilde{x})(u)-x_{0}\left(u_{0}-a_{0}\right)
$$

\footnotetext{
${ }^{12}$ Recall that $\tilde{x}$ does exist (Proposition 15).
} 
for $u \geq u_{0}$. Therefore, since $\rho$ is translation invariant and only the support of $\mathbb{P}$ affects $\rho$ and $\Pi$,

$$
\begin{aligned}
& \rho\left(J\left(\tilde{x}^{\prime}\right)\right)+e^{r T} \Pi\left(J\left(\tilde{x}^{\prime}\right)\right)= \\
& \rho\left(J(\tilde{x})+x_{0}\left(u_{0}-a_{0}\right)\right)+e^{r T} \Pi(J(\tilde{x}))-x_{0}\left(u_{0}-a_{0}\right) \\
& =\rho(J(\tilde{x}))+e^{r T} \Pi(J(\tilde{x})) .
\end{aligned}
$$

Hence, the objective of (33) attains an identical value for $x=\tilde{x}$ or $x=\tilde{x}^{\prime}$.

To sum up, we can consider that $\tilde{x}=\left(\tilde{x}_{1}, \tilde{x}_{2}, \ldots \tilde{x}_{m}\right)$ in the sense that $\tilde{x}(u)=$ $\tilde{x}_{j}$ for $u \in\left(u_{j-1}, u_{j}\right], j=1,2, \ldots, m$, and $\tilde{x}=0$ otherwise. According to (33), (38) and Theorem 18, the optimal $S G D\left(\tilde{x}_{1}, \tilde{x}_{2}, \ldots \tilde{x}_{m}\right)$ will be characterized by

$$
\begin{cases}\mathbb{E}(J(\tilde{x}) z) \geq \mathbb{E}(J(\tilde{x}) \tilde{z}), & \forall z \in \Delta_{\rho} \\ \left\langle\tilde{x}_{j}-1, \tilde{w}_{j}^{+}\right\rangle=\left\langle\tilde{x}_{j}+1, \tilde{w}_{j}^{-}\right\rangle=0, & j=1,2, \ldots, m\end{cases}
$$

As a particular case, the binomial model is very often used as an approximation of the $B S$ model. If $m$ is a natural positive number, then the time interval $[0, T]$ may be divided into $m+1$ subintervals $\left[t_{0}=0, t_{1}\right],\left[t_{1}, t_{2}\right], \ldots,\left[t_{m-1}, t_{m}=T\right]$ such that $t_{j}-t_{j-1}=T / m, j=1,2, \ldots, m$. Between $t_{j-1}$ and $t_{j}$ the value of $S$ may be multiplied by $e^{\sigma \sqrt{T / m}}>1$ or $e^{-\sigma \sqrt{T / m}}<1$, the physical $\eta_{p h}$ and the risk-neutral $\eta_{r n}$ probability of multiplying by $e^{\sigma \sqrt{T / m}}$ equal

$$
\eta_{p h}=\frac{e^{\mu(T / m)}-e^{-\sigma \sqrt{T / m}}}{e^{\sigma \sqrt{T / m}}-e^{\sigma \sqrt{T / m}}} \quad \eta_{r n}=\frac{e^{r(T / m)}-e^{-\sigma \sqrt{T / m}}}{e^{\sigma \sqrt{T / m}}-e^{\sigma \sqrt{T / m}}} .
$$

The set of the $m+1$ potential values of $S_{T}$ becomes

$U=\left\{S_{0} e^{-m \sigma \sqrt{T / m}}<S_{0} e^{(-m+2) \sigma \sqrt{T / m}}<S_{0} e^{(-m+4) \sigma \sqrt{T / m}} \ldots<S_{0} e^{m \sigma \sqrt{T / m}}\right\}$,

The probabilities in $U$ become

$$
\left\{\begin{array}{l}
\mathbb{P}\left(S_{T}=u_{j}=S_{0} e^{(-m+2 j) \sigma \sqrt{T / m}}\right)=\left(\begin{array}{c}
m \\
j
\end{array}\right) \eta_{p h}^{j}\left(1-\eta_{p h}\right)^{m-j} \\
\mathbb{Q}\left(S_{T}=u_{j}=S_{0} e^{(-m+2 j) \sigma \sqrt{T / m}}\right)=\left(\begin{array}{c}
m \\
j
\end{array}\right) \eta_{r n}^{j}\left(1-\eta_{r n}\right)^{m-j}
\end{array}\right.
$$

$j=0,1, \ldots, m$. If $\rho=C V @ R_{\beta}$ then (58) shows that (59) becomes

$$
\begin{cases}\operatorname{Min} \sum_{j=1}^{m}\left(u_{j}-u_{j-1}\right)\left(w_{j}^{+}+w_{j}^{-}\right) & \\
\sum_{k=j}^{m}\left(\begin{array}{c}
m \\
k
\end{array}\right) \eta_{p h}^{k}\left(1-\eta_{p h}\right)^{m-k} z_{k}-w_{j}^{+}+w_{j}^{-}=\mathbb{Q}\left(S_{T} \geq u_{j}\right), & j=1,2, \ldots, m \\
\sum_{j=1}^{m}\left(\begin{array}{c}
m \\
j
\end{array}\right) \eta_{p h}^{j}\left(1-\eta_{p h}\right)^{m-j} z_{j}=1 & \\
0 \leq z_{j} \leq 1 /(1-\beta) & j=0,1, \ldots, m \\
w_{j}^{+} \geq 0, w_{j}^{-} \geq 0, & j=1,2, \ldots, m\end{cases}
$$


This simple linear problem has a new dual that can be considered as the doubledual of (33). This double-dual becomes (Tamiz et al., 1998)

$$
\left\{\begin{array}{l}
\text { Max } \sum_{j=1}^{m}\left(u_{j}-u_{j-1}\right) \hat{\mathbb{P}}\left(S_{T} \geq u_{j}\right) \lambda_{j}+\lambda_{m+1}+\frac{1}{1-\beta} \sum_{j=m+2}^{2 m+2} \lambda_{j} \\
\mathbb{P}\left(S_{T}=u_{0}\right) \lambda_{m+1}+\lambda_{m+2} \leq 0 \\
\left(u_{1}-u_{0}\right) \mathbb{P}\left(S_{T}=u_{1}\right) \lambda_{1}+\mathbb{P}\left(S_{T}=u_{1}\right) \lambda_{m+1}+\lambda_{m+3} \leq 0 \\
\left\{\begin{array}{l}
\left(u_{1}-u_{0}\right) \mathbb{P}\left(S_{T}=u_{1}\right) \lambda_{1}+\left(u_{2}-u_{1}\right) \mathbb{P}\left(S_{T}=u_{2}\right) \lambda_{2}+ \\
\mathbb{P}\left(S_{T}=u_{2}\right) \lambda_{m+1}+\lambda_{m+4} \leq 0
\end{array}\right. \\
\ldots \begin{array}{l}
\left(u_{1}-u_{0}\right) \mathbb{P}\left(S_{T}=u_{m}\right) \lambda_{1}+\ldots+\left(u_{m}-u_{m-1}\right) \mathbb{P}\left(S_{T}=u_{m}\right) \lambda_{m}+ \\
\mathbb{P}\left(S_{T}=u_{m}\right) \lambda_{m+1}+\lambda_{2 m+2} \leq 0 \\
-1 \leq \lambda_{1}, \lambda_{2}, \ldots, \lambda_{m} \leq 1 \text { and } \lambda_{m+2}, \lambda_{m+3}, \ldots, \lambda_{2 m+2} \leq 0
\end{array}
\end{array}\right.
$$

$\lambda=\left(\lambda_{1}, \lambda_{2}, \ldots, \lambda_{2 m+2}\right)$ being the decision variable. Moreover, following a similar result of Balbás et al. (2015), if $\tilde{\lambda}=\left(\tilde{\lambda}_{1}, \tilde{\lambda}_{2}, \ldots, \tilde{\lambda}_{2 m+2}\right)$ solves (62), then $\tilde{x}=$ $\left(\tilde{\lambda}_{1}, \tilde{\lambda}_{2}, \ldots, \tilde{\lambda}_{m}\right)$ solves (33), and therefore (61) and (62) allow us to find both optimal solutions $\left(\tilde{z}_{0}, \ldots, \tilde{z}_{m}, \tilde{w}_{1}^{+}, \ldots, \tilde{w}_{m}^{+}, \tilde{w}_{1}^{-}, \ldots, \tilde{w}_{m}^{-}\right)$and $\left(\tilde{x}_{1}, \tilde{x}_{2}, \ldots \tilde{x}_{m}\right)$.

\section{Conclusion}

Smooth self-financing strategies with negative risk $(S G D)$ frequently exist and may be easily constructed. If the selected risk measure is $\rho=V @ R$ then the $S G D$ is just the sale of a "golden European option" along with the investment of the option price in a riskless asset. The strike of the option is easy to estimate, and the existence of this option does not require particular assumptions. Actually, one can involve risks with both bounded and unbounded expectation, as well as friction-free and non-friction-free pricing rules. Numerical experiments have been reported.

If $\rho$ is a coherent and expectation bounded risk measure then the framework has to be more limited, but it is still general enough. Actually, expectations and variances have to remain finite, and the pricing rule $\Pi$ must be continuous, but $\Pi$ may still incorporate potential frictions. Under this setting the existence of $S G D s$ has been characterized by means of practical conditions. If $\rho=C V @ R$ then the optimal $S G D$ is composed of the sale of a "golden European put" along with the investment of the put price in a riskless asset. Numerical experiments have been reported.

Though computational issues have been properly addressed, it has been also shown that they become really simple in a discrete framework, since only finitedimensional linear programming methods have to be involved. Discrete models are interesting for both reasons, they are simple and the usual pricing models often have a good enough discrete approximation. 
Acknowledgments. This research was partially supported by the University Carlos III of Madrid (Project 2009/00445/002) and the Spanish Royal Academy of Sciences. The usual caveat applies.

\section{References}

Anderson, E.J. and P. Nash (1987). Linear programming in infinite-dimensional spaces. John Wiley \& Sons.

Assa, H. (2016). Natural risk measures. Mathematics and Financial Economics, 10, 441 - 456.

Artzner, P., F. Delbaen, J.M. Eber and D. Heath (1999). Coherent measures of risk. Mathematical Finance, 9, $203-228$.

Balbás, A., B. Balbás and R. Balbás (2016). Outperforming benchmarks with their derivatives: Theory and empirical evidence. Journal of Risk, 18, 4, $25-52$.

Balbás, A., B. Balbás and R. Balbás (2017). Differential equations connecting $V a R$ and $C V a R$. Journal of Computational and Applied Mathematics, 326, $247-267$.

Balbás, A., B. Balbás, R. Balbás and A. Heras (2015). Optimal reinsurance under risk and uncertainty. Insurance: Mathematics and Economics, 60, 61 74 .

Balbás, A., J. Garrido and S. Mayoral (2009). Properties of distortion risk measures. Methodology and Computing in Applied Probability, 11, 385 - 399.

Bernardo, A.E. and O. Ledoit (2000). Gain, loss, and asset pricing. Journal of Political Economy, 108, 144 - 172.

Biagini, S. and M.C. Pinar (2013). The best gain-loss ratio is a poor performance measure. SIAM Journal of Financial Mathematics, 4, 228 - 242.

Bondarenko, O. (2014). Why are put options so expensive? Quarterly Journal of Finance, 4, 3, 1450015.

Cheung, K.C., W.F. Chong and S.Yam (2015). Convex ordering for insurance preferences. Insurance: Mathematics and Economics, 64, 409 - 416.

Cochrane, J. H., and J. Saa-Requejo (2000). Beyond arbitrage: good deal asset price bounds in incomplete markets. Journal of Political Economy, 108, $79-119$

Duffie, D. (1988). Security markets: Stochastic models. Academic Press.

Filipović, D., M. Kupper and N. Vogelpoth (2012). Approaches to conditional risk. SIAM Journal of Financial Mathematics, 3, 402 - 432.

Jarrow, R., and M. Larsson (2012). The meaning of market efficiency. Mathematical Finance, 22, 1 - 30.

Jouini, E., M. Meddeb and N. Touzi (2004). Vector-valued coherent risk measures. Finance and Stochastics, 8, 531 - 552.

Konstantinides, D.G. and G.C. Zachos (2018). Exhibiting abnormal returns under a risk averse strategy. Methodoloygy and Computing in Applied Probability, forthcoming, https://doi.org/10.1007/s11009-018-9673-9.

Kopp, P.E. (2008). Martingales and stochastic integrals. Cambridge University Press. 
Kupper, M., and W. Schachermayer (2009). Representation results for law invariant time consistent functions. Mathematics and Financial Economics, 2, $189-210$.

Luenberger, D.G. (1969). Optimization by vector spaces methods. John Wiley \& Sons.

Mausser, H., D. Saunders and L. Seco (2006). Optimizing omega. Risk Magazine, 11, 88 - 92.

Pichler, A. (2014). Insurance pricing under ambiguity. European Actuarial Journal, 4, 335 - 364 .

Rockafellar, R. T., S. Uryasev and M. Zabarankin (2006). Generalized deviations in risk analysis. Finance and Stochastics, 10, $51-74$.

Salas-Molina, F. (2018). Selecting the best risk measure in multiobjective cash management. International Transactions in Operational Research, forthcoming, https://doi.org/10.1111/itor.12580.

Schaeffer, H.H. (1970). Topological vector spaces. Springer.

Schaeffer, H.H. (1974). Banach lattices and positive operators. Springer.

Tamiz, M., D. Jones and C. Romero (1998). Goal programming for decision making: An overview of the current state-of-the-art. European Journal of Operational Research, 111, 569 - 581.

Zhao, P. and Q. Xiao (2016). Portfolio selection problem with Value-at-Risk constraints under non-extensive statistical mechanics. Journal of Computational and Applied Mathematics, 298, 74 - 91. 